\title{
Präklinische Versorgung des Polytraumas
}

\author{
Delovan Oramary, Onays Al-Sadi, Sophia Blum, Klaus Dieter Schaser, Christian Kleber
}

\section{Einleitung}

Das Trauma stellt nach wie vor eine der häufigsten Todesursachen des jungen Erwachsenen dar. Durch Verbesserungen in der Unfallprävention und bei Fahrzeugsicherheitssystemen konnte die Inzidenz an Unfalltoten in den letzten Jahrzehnten deutlich reduziert werden. Genaue statistische Erfassungen von Unfalltoten (Arbeitsunfälle, Freizeitunfälle, Verkehrsunfälle) existieren in Deutschland aktuell leider nicht. Verbesserungen des klinischen Managements (z. B. ATLS ${ }^{\circledR}$, ETC $^{\circledR}$, Damage Control Surgery, Traumanetzwerke) und der intensivmedizinischen Therapie mit Organersatzverfahren führten zu einer deutlichen Reduktion der Klinikletalität. Eine systematische Erfassung der Traumasterblichkeit in Berlin 2010 konnte die Präklinik mit 59\% der Verstorbenen als Haupttodesort und die Zeitphase < 60 min nach Trauma (57\%) als den Haupttodeszeitpunkt nachweisen [1]. Somit rückt die präklinische Polytraumaversorgung weiter in den Fokus. Dem entgegen steht die Tatsache, dass der traumatologische Notfall- oder Notarzteinsatz eine zunehmende Rarität darstellt (ca. 1\% der Notarzteinsätze). In Abhängigkeit des Rettungsmittels (luft-/bodengebunden) sind dies zwischen 1 und $20 \%$ der Einsätze. Beispielhaft hat ein bodengebundener Notarzt durchschnittlich alle 15 Monate ein Polytrauma zu versorgen. Somit besteht ein Spannungsfeld aus Notwendigkeit von Erfahrung und manuellen Fähigkeiten mit invasiven Rettungstechniken (z.B. Thoraxdrainage) einerseits und mangelnder Erfahrung und Ausbildung andererseits.

Im folgenden Artikel werden aktuelle Leitlinien und Algorithmen zur präklinischen Versorgung des Polytraumas vorgestellt.

\section{Definition}

Bei einem Polytrauma handelt es sich laut Definition von $H$. Tscherne um eine Verletzung von mehreren Körperregionen oder Organsystemen, wobei wenigstens eine dieser Verletzungen oder die Kombination mehrerer Verletzungen lebensbedrohlich ist. Klinische oder wissenschaftliche Klassifikation wie der Injury Severity Score (ISS) oder Abbreviated Injury Severity Score (AIS) finden präklinisch aufgrund der fehlenden radiologischen Diagnostik keine Anwendung. In Abhängigkeit von der physiologischen Verletzungsschwere können Polytraumapatienten jedoch auch präklinisch in 3 wesentliche Gruppen eingeteilt werden ( $\bullet$ Tab. 1$)$.

Obige Klassifikation des Patienten macht auch im Rahmen des präklinischen Managements Sinn, da dies einen unmittelbaren Einfluss auf die Einsatztaktik hat. Wohingegen es keinen linearen Zusammenhang zwischen der Länge der Rettungszeit und nachfolgender Klinikletalität im deutschen Notarztsystem gibt, existiert selbstverständlich eine Untergruppe, die „instabilen/in extremis“ Patienten mit mutmaßlichen schweren inneren Blutungen, die von einer „Treat-and-Run“-Strategie profitieren [2].

\section{Epidemiologie}

Das TraumaRegister DGU ${ }^{\circledR}$ (TR-DGU) liefert allein in Deutschland mit einer Teilnahme von ca. 700 Kliniken eine nahezu flächendeckende Dokumentation der im Krankenhaus versorgten Schwerverletzten in Deutschland (> 270000 Fälle). Dabei war der typisch polytraumatisierte Patient im Jahresbericht 201852 Jahre alt und in $70 \%$ der Fälle männlich.

Fast die Hälfte aller registrierten Schwerverletzten (49\%) verunglückten im Straßenverkehr (Pkw 21\%, Motorrad

- Tab. 1 Klinische Klassifikation der physiologischen Verletzungsschwere eines Polytraumas.

\begin{tabular}{|l|l|l|l|}
\hline & stabil & Borderline & instabil/in extremis \\
\hline systolischer Blutdruck & $>100 \mathrm{mmHg}$ & $80-100 \mathrm{mmHg}$ & $<80 \mathrm{mmHg}$ \\
\hline Veränderung Blutdruck nach Volumengabe* & $\uparrow \uparrow$ & $\uparrow \downarrow$ & $-\downarrow$ \\
\hline * Applikation von $500-1000 \mathrm{ml}$ kristalloider Infusionslösung und Reevaluation des systolischen Blutdruckes & \\
\hline
\end{tabular}


- Tab. 2 Kumulativer innerer Blutverlust bei geschlossenen Frakturen [9].

\begin{tabular}{|l|c|}
\hline Humerus & $100-800 \mathrm{ml}$ \\
\hline Radius/Ulna & $50-400 \mathrm{ml}$ \\
\hline Becken/Femur & $500-5000 \mathrm{ml}$ \\
\hline Tibia & $100-1000 \mathrm{ml}$ \\
\hline
\end{tabular}

13\%, Fahrradfahrer 9\%, Fußgänger 6\%), 41\% Stürze und $10 \%$ andere Unfallursachen. Die häufigsten betroffenen Körperregionen waren dabei der Kopf (48\%), Thorax (45\%) und Wirbelsäule (29\%) gefolgt von Verletzungen der oberen (28\%) bzw. unteren Extremitäten (24\%), des Beckens (15\%) und Bauches (14\%) [3].

Das Polytrauma gehört jedoch mit ca. $1 \%$ zu den selteneren Notarzteinsätzen, wohingegen $57 \%$ der Patienten innerhalb der 1. Stunde nach Trauma versterben, weshalb in dieser vulnerablen Phase die optimale Einleitung lebensrettender Maßnahmen, das richtige Zeitmanagement (Einsatztaktik) und der rasche Transport in ein geeignetes Traumazentrum wichtige Kernelemente für ein erfolgreiches Management darstellen. Speziell in der präklinischen Phase sind anhand einer prospektiven Beobachtungsstudie Situationen bekannt, in denen präklinische Maßnahmen zur Reduzierung von vermeidbaren Todesfällen ( $5 \%$ definitiv, $10 \%$ potenziell vermeidbar) führen können. Wesentliche Probleme zeigten sich bei der Atemwegssicherung, der adäquaten Entlastung eines Spannungspneumothoraxes, bei der externen Beckenstabilisation oder suffizienten Blutungskontrolle $[1,4]$.

\section{Einsatzgrundsätze}

Das Polytrauma stellt entsprechend der Empfehlung der Bundesärztekammer von 2013 einen Notarzteinsatz dar [5]. Beim Eintreffen soll zunächst der Unfallmechanismus, die vorliegende Umgebungssituation, die Anzahl der betroffenen Personen sowie das Ausmaß des Traumas eingeschätzt werden. Dabei ist der nötige Eigenschutz in Gefahrensituationen und somit der Ausschluss von Eigen- bzw. Fremdgefährdung besonders wichtig. In Situationen mit mehreren Verletzten oder sogar Massenanfall von Verletzten (MANV) ist die Funktion des ersteintreffenden Notarztes die des Leitenden Notarztes. Hierbei haben organisatorische und einsatztaktische Aufgaben Priorität gegenüber der individualmedizinischen Behandlung eines Patienten.

Wesentliche Aufgaben des Notarztes ist die Sicherung und Aufrechterhaltung der Vitalfunktionen, Linderung von Schmerzen, Herstellung der Transportfähigkeit, Begleitung und Überwachung des Patienten bis zur Einlieferung in eine geeignete Klinik. Hierbei sollte dem Notarzt das Leistungsspektrum der Kliniken entsprechend den
Kriterien des TraumaNetzwerks DGU ${ }^{\circledR}$ in seinem Einsatzgebiet bekannt sein.

Die Luftrettung soll primär zur Versorgung Schwerverletzter eingesetzt werden. Einsatztaktische Gesichtspunkte und der Zeitfaktor sind zu berücksichtigen [6].

\section{Strukturiertes und prioritäten- orientiertes Traumamanagement (ABCDE-Schema)}

Anlässlich der Komplexität eines Polytraumas mit multiplen Verletzungskombinationen und teilweise simultanen lebensbedrohlichen Problemen hat sich das ABCDE-Schema der Notfallmedizin als Grundlage der präklinischen Polytraumaversorgung in Rahmen von Kurskonzepten (z. B. pHTLS ${ }^{\circledR}$ [PreHospital Trauma Life Support], ETC ${ }^{\circledR}$ [European Trauma Course], TraumaManagement ${ }^{\circledR}$ ) durchgesetzt. Neben einer strukturierten Erfassung der medizinischen Probleme und Verletzungen geben die Algorithmen auch die Priorität der therapeutischen Maßnahmen vor. Der Gesamtumfang der notärztlichen Versorgung hängt neben dem individuellen Patientenzustand auch von den Umgebungsbedingungen (Witterung, Lichtverhältnisse, Entfernung Klinik) ab. Prinzipiell sollen alle notwendigen Maßnahmen zur Wiederherstellung und Aufrechterhaltung der Vitalfunktionen unter Berücksichtigung einer möglichst kurzen Rettungszeit durchgeführt werden („Treat and Run“). Große innere Blutungen hingegen können präklinisch nicht beherrscht werden (nicht kompressible Blutungen) und können von einer „Load-and-Go“-Strategie profitieren [7].

Im Zuge des schweren Polytraumas stehen pathophysiologische Probleme wie Hypovolämie, Blutung, Azidose, Asphyxie, Koagulopathie und Dysregulation des Immunsystems (SIRS, Sepsis) im Vordergrund, die bei nicht adäquater Therapie zum Multiorganversagen und Versterben des Patienten führen [8]. Die Präklinik ist vor allem durch die 2 wesentlichen Problemkomplexe „Hypovolämie/Schock/Koagulopathie“ und „Asphyxie/Hypoxie“ geprägt.

Relevante Blutungsquellen stellen dabei Thorax, Abdomen, Becken, große Röhrenknochen und externe Blutungen dar. Auch die Summation von Einzelverletzungen, z.B. Frakturen der oberen und unteren Extremitäten, können zu einem lebensbedrohlichen kumulativen Blutverlust führen ( Tab. 2) [9].

Nahezu alle Blutungslokalisationen können durch die körperliche Untersuchung auch präklinisch eingeschätzt werden. Die Suche nach Prellmarken, Schürfwunden („Seat Belt Sign“) und die Bewertung des Unfallmechanismus lassen dabei gute Rückschlüsse auf die mutmaßli- 
chen Verletzungen zu. Ansprechbare Patienten sollen nach aktuellen Beschwerden und deren Lokalisation befragt werden. Eine frühzeitige sowie bedarfsgerechte Analgesie ist in den meisten Fällen erforderlich. Zur Beurteilung des Schweregrades der Verletzungen ist eine präklinische Untersuchung am Unfallort notwendig, allerdings erfolgt diese in angebrachtem Maße und sollte die Gesamtrettungszeit nicht wesentlich verzögern. Die Gesamtdauer der Untersuchung sollte nicht länger als 5 min betragen und erfolgt orientierend vom Kopf bis zum Fuß [6].

Die Reihenfolge der Untersuchung sollte dabei folgende Aspekte berücksichtigen: Inspektion (Fehlstellung/Wunden/Schwellung/Durchblutung), Stabilitätsprüfung (Krepitation, abnorme Beweglichkeit, sichere und unsichere Frakturzeichen), Beurteilung der Durchblutung, Motorik und Sensibilität. Das häufig begleitende Weichteiltrauma sollte ebenso beurteilt werden (geschlossene vs. offene Fraktur, Hinweise auf Kompartmentsyndrom). An den Extremitäten soll im Seitenvergleich die kapillare Reperfusion getestet werden [6]. Zusätzlich zur körperlichen Untersuchung erfolgt die Erhebung der Vitalparameter. Diese beinhaltet die Ermittlung der Atemfrequenz (AF), die Auskultation, Messung des Blutdruckes sowie der Sauerstoffsättigung $\left(\mathrm{SpO}_{2}\right)$. Das Basismonitoring besteht aus oszillierender Blutdruckmessung, Pulsoxymetrie, 3Kanal-EKG und Kapnometrie bei Beatmung. Trotz obig genannter Techniken und Möglichkeiten ist die präklinische Diagnostik limitiert und fehlerhaft. Es wurden Fälle berichtet, bei denen ein zugrunde liegendes Polytrauma vom Rettungsteam gar nicht erkannt wurde $[1,4]$. Hierbei sind die Bewertung der Notfallsituation und die körperliche Untersuchung geeignete Instrumente. Dennoch sind auch in der Rechtsmedizin Fälle von polytraumatisierten Patienten beschrieben, die äußerlich vollständig unversehrt anmuten (Casper-Zeichen) [10,11]. Hierbei ist eine äußerliche körperliche Untersuchung ggf. auch nicht hinweisgebend. Wird eine Verletzung festgestellt, werden die Schwere der Verletzung und der vorliegende Blutverlust meist falsch eingeschätzt. Leichte Verletzungen oder niedrige Blutverluste werden über- und schwere Verletzungen oder hohe Blutverluste unterschätzt [12]. Ähnliche Schwierigkeiten weist die klinische Untersuchung des Beckens auf. In der Hand des Geübten hat sie eine hohe Sensitivität, wohingegen der Ungeübte meist zu Fehleinschätzungen neigt $[13,14]$. Diesbezüglich wird die generelle Empfehlung der Beckenuntersuchung kritisch diskutiert [5]. In der Praxis hat sich das Vorgehen der präventiven Stabilisation und Behandlung analog der HWS-Immobilisation bewährt.

\section{A: Atemweg sichern und HWS-Immobilisation}

Die Immobilisierung der Halswirbelsäule (HWS) manuell bzw. mittels Zervikalstütze sollte präklinisch als primäre Maßnahme für einen Unfallverletzten erfolgen.
Störungen des Atemwegs können im Rahmen von Schädel-Hirn-Traumata mit quantitativer Vigilanzminderung, durch direktes Trauma gegen den Hals/Kehlkopf und Mittelgesichtsverletzungen bedingt sein. Verletzungen der oberen HWS können zu einer Atemwegsverlegung sowie zu Myelonschäden führen, wodurch eine Atemdepression bis hin zum Atemstillstand resultieren kann.

Die endotracheale Intubation stellt den Goldstandard zur präklinischen Atemwegssicherung dar $[15,16]$. Die korrekte Intubation ist ein Grundpfeiler der invasiven präklinischen Maßnahmen und unmittelbar mit dem Überleben assoziiert [17]. Allerdings bestehen in der prähospitalen Phase in Hinblick auf den Intubationserfolg signifikante Unterschiede zwischen geübtem und ungeübtem ärztlichen Personal, weshalb ein regelmäßiges Training der Notfallnarkose, der endotrachealen Intubation und der alternativen Methoden zur Atemwegssicherung (Maskenbeatmung mit/ohne Guedel-/Wendeltubus, supraglottische Atemwegshilfen, Notfallkoniotomie) empfohlen werden $[6,18]$. Alternativen stellen die supraglottischen Atemwegshilfen wie Wendel- und Guedel-Tubus im Rahmen der Maskenbeatmung, der Larynx-/Kombitubus oder die Larynxmaske dar. Im Rahmen von schweren Mittelgesichtsverletzungen oder einer „Cannot-intubate-cannot-ventilate“-Situation kann auch der chirurgische Atemweg mit Cricothyroidotomie, Koniotomie oder Tracheotomie notwendig sein. Aus Sicht der Autoren hat sich hier die Cricothyroidotomie in SeldingerTechnik als sichere und zuverlässige Methode durchgesetzt ( $\triangleright$ Abb. 1) [19].

Indikationen zur Atemwegssicherung bei polytraumatisierten Patienten bestehen bei Apnoe oder Schnappatmung (Atemfrequenz $[\mathrm{AF}]<6 / \mathrm{min} />29 / \mathrm{min}$ ) und quantitativer Vigilanzminderung (Glasgow Coma Scale [GCS] <9 Punkte) [6] sowie bei einem geplanten Transport mit dem Rettungshubschrauber.

Die prähospitale Atemwegssicherung erfolgt aufgrund des Aspirationsrisikos und der meist fehlenden Nüchternheit des polytraumatisierten Patienten als „RapidSequence“-Intubation, wobei eine Kombination aus Analgetikum (z. B. Fentanyl), Sedativum (z. B. Midazolam) und Muskelrelaxans (z.B. Succinylcholin) angewendet wird [20]. Bedingt durch die verbundenen Nebenwirkungen auf die Nebennierenfunktion soll Etomidat als Einleitungshypnotikum vermieden werden. Ketamin eignet sich hierbei als gute Alternative [6].

Vor der Narkoseeinleitung soll der spontan atmende Patient, wann immer vertretbar, bis zu 4 min mit 100\% Sauerstoff via Gesichtsmaske mit Reservoir präoxygeniert werden [6]. Während der Intubation wird die Halswirbelsäule mithilfe der „manuellen Inline-Stabilisierung“ (MILS) unter temporärer Entfernung der HWS-Orthese stabilisiert [5]. Nach mehr als 2 Intubationsversuchen sollen al- 


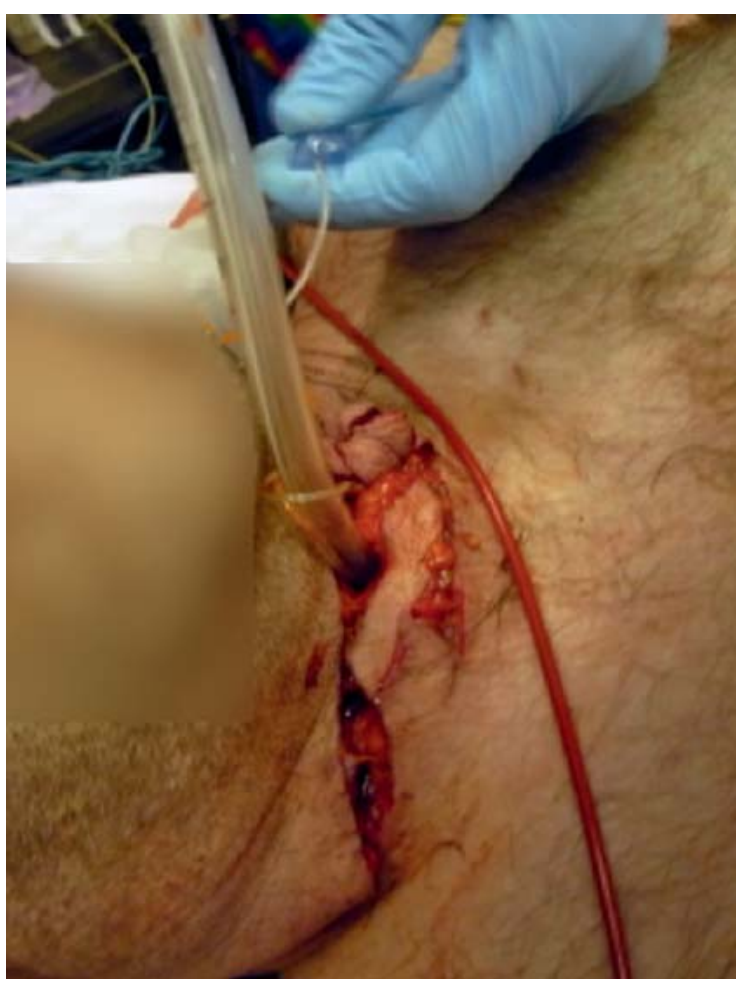

- Abb. 1 Extremform einer alternativen Atemwegssicherung: M56], Schnittverletzung am Hals in suizidaler Absicht mit einem Küchenmesser. Direkte Insertion des Beatmungstubus in die freiliegende Trachea.

ternative Methoden zur Atemwegssicherung (siehe oben) in Betracht gezogen werden. Die Videolaryngoskopie stellt eine gute Option zur Verbesserung der Einstellbarkeit der Stimmbandebene dar und sollte deshalb auch in der Präklinik auf notarztbesetzten Einsatzmitteln vorgehalten werden [5].

Der einzig klinisch wie auch forensisch sichere Nachweis der korrekten Tubuslage stellt präklinisch die Kapnometrie $($ etCO 2 ) dar [6]. Weiterhin kann die Kapnometrie zur Steuerung der Beatmung, Ausschluss einer Tubusdislokation bei Rettungs- und/oder Umlagerungsmaßnahmen und indirekten Einschätzung der Kreislaufsituation (vor allem bei Patienten mit permissiver Hypotonie; siehe C) eingesetzt werden. Bei Patienten mit Vitae minimae kann aufgrund der Zentralisation meist peripher kein Blutdruck mehr gemessen werden, wobei die et $\mathrm{CO}_{2}$-Kurve eine Beurteilung zulässt. Dies trifft auch auf die erfolgreiche Postreanimationsphase zu.

\section{B: Be-/Atmung}

Die klinische Untersuchung des Thoraxes sowie die Überprüfung der regelrechten Atemfunktion stellt die Basis zur Einschätzung der Atmung dar. Dabei empfiehlt es sich, die Atemfrequenz zu bestimmen und die Lungen zu auskultieren. Im Rahmen einer fokussierten Untersu- chung können zusätzlich die Inspektion, Palpation und limitiert auch Perkussion auf thorakale Verletzungen hinweisen. Zusätzlich kommen technische Hilfsmittel wie die $\mathrm{SpO}_{2}$ - und et $\mathrm{CO}_{2}$-Messung zur Anwendung. Patienten mit suffizienter Spontanatmung können unterstützend Sauerstoff via Nasenbrille oder Maske erhalten. Die Indikation zur Notfallnarkose, endotrachealen Intubation und suffizienter Beatmung besteht dabei bei

- Hypoxie $\left(\mathrm{SpO}_{2}<90 \%\right)$ trotz adäquater Sauerstoffgabe und ohne klinisch vorliegenden Spannungspneumothorax

- schweres Schädel-Hirn-Trauma (SHT; GCS < 9 Punkte)

- traumaassoziierte, persistierende hämodynamische Instabilität $\left(R_{\text {sys }}<90 \mathrm{mmHg}\right.$, altersadaptiert bei Kindern)

- schweres Thoraxtrauma mit respiratorischer Insuffizienz (AF > 29/min, altersadaptiert bei Kindern)

Nach erfolgreicher Intubation (siehe A) wird eine Normoventilation ( $\mathrm{etCO}_{2} 35-45 \mathrm{mmHg} ; \mathrm{SpO}_{2}>90 \%$ ) angestrebt [6]. Vor Indikationsstellung zur Notfallnarkose sollten selbstverständlich reversible Ursachen einer Hypoventilation wie der Spannungspneumothorax ausgeschlossen werden.

\section{Spannungspneumothorax}

Das schwere Thoraxtrauma führt überdurchschnittlich häufig zu einer ausgeprägten respiratorischen Insuffizienz (31\%) oder ist mit einem Kreislaufversagen (26\%) vergesellschaftet. Die Sterblichkeit bei signifikantem Thoraxtrauma liegt bei etwa $10 \%$. Mit zunehmender Schwere des Thoraxtraumas steigt die Letalität und beträgt bei einem AIS von 5 Punkten im Mittel um 30\%. Der Spannungspneumothorax stellt präklinisch einen akut lebensbedrohlichen Zustand dar, der ohne sofortige Entlastung in aller Regel tödlich endet. Er stellt die häufigste reversible Ursache des traumatischen Herz-Kreislauf-Stillstands dar und muss in der Präklinik entlastet werden $[1,5]$.

Bis zu $25 \%$ aller polytraumatisierten Patienten versterben an einem schweren Thoraxtrauma, obwohl bis zu $90 \%$ mittels Anlage einer einfachen Saugdrainage erfolgreich behandelt werden könnten! [1,5].

Hinweisgebend auf einen Spannungspneumothorax sind:

- abgeschwächtes Atemgeräusch in Auskultation

- Weichteilemphysem (klinische Untersuchung)

- instabiler Thorax (klinische Untersuchung)

- obere Einflussstauung (Halsvenen)

- eFAST (Wiederholungsechos)

Bei einseitig abgeschwächtem oder fehlendem Atemgeräusch (nach Kontrolle der korrekten Tubuslage) soll die Verdachtsdiagnose Pneumo- und/oder Hämatothorax gestellt werden. Das Fehlen eines solchen Auskultationsbefundes, insbesondere bei Normopnoe und thorakaler Schmerzfreiheit, schließt einen größeren Pneumothorax 
weitgehend aus. Ein Hautemphysem ist nahezu beweisend für das Vorliegen eines Pneumothoraxes.

Kleine, klinisch nicht diagnostizierbare Pneumothoraces, speziell bei spontan atmenden Patienten, sind selten progredient und benötigen keine notfallmäßige Dekompression in der Präklinik. Dennoch kann es zu einer Progression eines zuvor klinisch inapparenten Pneumothoraxes kommen [6]. Typisch hingegen ist ein Pneumothorax, der nach notwendiger Intubation und Beatmung (Überdruck In- und Exspiration) diesen in einen Spannungspneumothorax überführt. In Abhängigkeit vom Beatmungsmodus ist dies neben den klinischen Symptomen durch Überdruckalarm (volumenkontrollierte Beatmung) oder niedriges Tidalvolumen/Atemminutenvolumen (druckkontrollierter Beatmung) assoziiert.

Zur pleuralen Dekompression stehen dabei 2 wesentliche Verfahren zur Verfügung: die Entlastungspunktion im 2.3. Interkostalraum medioklavikular (Monaldi-Zugang) oder die Minithorakotomie mit Anlage einer Thoraxdrainage im 4.-5. Interkostalraum (Bülau-Position) der vorderen Axillarlinie. Zur Orientierung kann hier die Höhe der Mamille helfen, wobei dies bei Frauen als unzuverlässig einzustufen ist. Applikationen zu distal und Anwendung eines Trokars ohne palpatorische Lagekontrolle sind dabei wesentlich für Fehllagen (abdominell, pulmonal, kardial) ( $\bullet$ Abb. 2).

Bei Erwachsenen wird die Verwendung von 24-32French-Drainagen empfohlen. Die Anlage erfolgt unter sterilen Kautelen. Bei wachen Patienten wird eine Lokalanästhesie des Punktionsareals bis einschließlich der Pleura parietalis appliziert. Mit dem Skalpell erfolgt nun eine horizontale (parallel zur Rippe) ca. 4-5 cm lange Hautinzision. Nach stumpfer Präparation der Subkutis (Schere; Finger) und Interkostalmuskulatur am Oberrand der Rippe (da am Unterrand der Rippen Vene, Arterie und Nerv in den Interkostalraum ziehen) erfolgt anschließend die Eröffnung der Pleura mittels Finger. Nun wird durch Palpation der Lunge und Thoraxwand die korrekte Lage im Pleuraraum verifiziert und ggf. Adhäsionen gelöst. Zuletzt wird die Thoraxdrainage unter palpatorischer Kontrolle eingeführt und durch Pflasterzügel oder eine Annaht (wie z. B. die Tabaksbeutelnaht) gesichert [6]. Bei spontan atmenden Patienten kommt nun ein HeimlichVentil zur Kanalisation des Flusses zum Einsatz. Beim beatmeten Patienten ist dies aufgrund des unphysiologischen Überdruckes im Pleuraraum auch bei Inspiration nicht notwendig, sodass entweder spezielle Thoraxdrainagebeutel, Magensondenbeutel oder im Notfall eine Absaugpumpe mit reduziertem Druck angeschlossen werden können. Die Fördermenge der Thoraxdrainage sollte streng im Auge behalten werden und kann hinweisgebend auf die führende Blutungsquelle (Thorax) des Patienten sein. Bei thorakalen Blutungen unter Beatmung sollten moderate PEEP-Werte (5-10 $\mathrm{cm} \mathrm{H}_{2} \mathrm{O}$; PEEP: posi-

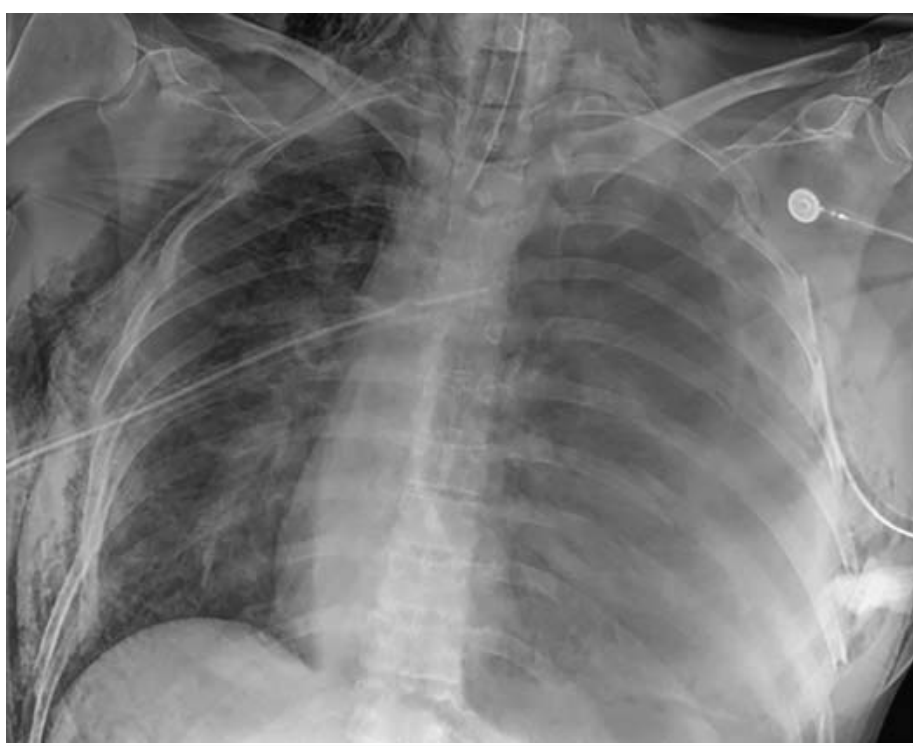

- Abb. 2 Radiologischer Nachweis einer insuffizienten Dekompression eines Pneumothoraxes mit massivem Weichteilemphysem bei Fehllage einer Thoraxdrainage. Quelle: Institut und Poliklinik für Diagnostische und Interventionelle Radiologie, Universitätsklinikum Carl Gustav Carus Dresden.

tiver endexspiratorischer Druck) angewendet werden, da ein hoher intrathorakaler Druck zur Aggravation von intrathorakalen, vor allem venösen Blutungen führen kann. Thorakale Blutungen stellen hierbei einen zeitkritischen Einsatz dar.

Offene Thoraxverletzungen führen meist zur respiratorischen Dekompensation mit Notwendigkeit einer Beatmung. In diesem Fall sollte ein nicht okklusives „Pleurasiegel“ angewendet werden, was bei mutmaßlicher pleuraler Verletzung das Entweichen der Luft zulässt. Bei spontan atmenden Patienten muss die offene Thoraxverletzung zur Vermeidung eines Medialstinalflatterns und damit respiratorischen Insuffizienz ebenfalls semiokklusiv abgedeckt werden [21]. Eine besondere Bedeutung bekommt die Anlage einer Thoraxdrainage bei einer geplanten Luftrettung. Ein übersehener und in dubio nicht adressierter Pneumothorax kann sich durch die Intubationsnarkose mit Überdruckbeatmung in der Luft zu einem Spannungspneumothorax entwickeln. Dies führt zu einer akut lebensbedrohlichen Situation, die zu einer Notfalllandung zwingt und beherbergt erhebliches Gefahrenpotenzial für den gesamten weiteren präklinischen Verlauf.

\section{C: Kreislauf}

Die im Rahmen eines Polytraumas eintretende Kreislaufinstabilität ist in den meisten Fällen durch eine Hypovolämie bedingt. Die führenden Blutungsquellen und deren präklinische Möglichkeit der Diagnostik wurden bereits zuvor abgehandelt. Die präklinische Sonografie kann hierbei zum Nachweis freier intraabdomineller Flüssigkeit 
- Tab. 3 Klassifikation und klinische Einschätzung des Blutverlustes nach Bolus-Applikation von 500-1000 ml kristalloider Infusionslösung mit jeweiliger Einsatztaktik.

\begin{tabular}{|l|l|l|l|}
\hline Responder & RR $\uparrow$ & kein relevanter weiterer Blutverlust & „Treat and Go“ \\
\hline temporärer Responder & RR $\uparrow$ & aktive Blutung & zeitkritisch \\
\hline Nonresponder & RR $-\downarrow$ & schwere aktive Blutung & "Treat/Scoop and Run“ \\
\hline
\end{tabular}

und damit Zuweisung einer entsprechenden Einsatztaktik dienen. Stark blutende Verletzungen der Extremitäten, die zu einer Beeinträchtigung der Vitalfunktion führen, sollen mit Priorität behandelt werden [5]. Klassische Symptome wie Tachykardie, Hypotonie, kaltschweißige, blasse Haut sowie eine verzögerte kapilläre Reperfusion (>2 s) deuten auf ein Schockgeschehen hin. Diesbezüglich kann trotz Einschränkungen der Schockindex (Quotient aus Herzfrequenz und $R_{\text {sys }}$ ) zur Orientierung für den vorliegenden Schweregrad des Schocks dienen [7].

Bei Verdacht auf innere Blutungen (Thorax, Abdomen, Becken) ist der schnellstmögliche Transport in eine geeignete Zielklinik zur chirurgischen Blutstillung erforderlich. Zur Reduzierung vor allem von inneren Blutungen hat sich insbesondere bei isolierten parenchymatösen Organverletzungen die sog. „permissive Hypotension“ ( $R R_{\text {sys }}$ $70-80 \mathrm{mmHg}$, mittlerer arterieller Druck $>50 \mathrm{mmHg}$ ) bewährt [6]. Diese ist bei Neurotraumata (SHT, Querschnitt) zugunsten eines suffizienten zerebralen und spinalen Perfusionsdruckes kontraindiziert [5].

Die Voraussetzung für eine ausreichende Gewebeperfusion zur adäquaten Volumentherapie ist die Anlage von großlumigen i.v. Zugängen. Studien zeigten, dass die primäre Applikation von Katecholaminen bei Hypovolämie im Vergleich zur Volumentherapie mit einer erhöhten Sterblichkeit assoziiert war. Somit sollten Katecholamine zur Aufrechterhaltung eines suffizienten Kreislaufes erst nach Versagen der Volumentherapie eingesetzt werden. Ein unreflektierter Einsatz von Infusionslösungen hingegen war ebenfalls mit einer erhöhten Inzidenz an Koagulopathie, Organversagen und Letalität assoziiert, sodass das Kreislaufmanagement beim polytraumatisierten Patienten differenziert erfolgen muss („Small Volume Rescucitation“) [6].

Im eigenen Vorgehen werden 2 großlumige i.v. Zugänge angelegt und bei Hypovolämie 500-1000 ml Kristalloid verabreicht. Anschließend erfolgt die Reevaluation der Kreislaufsituation mit Einstufung in nachfolgende Kategorien ( Tab. 3 ).

Bei der Wahl des Zugangs sollte darauf geachtet werden, dass diese nicht distal einer mutmaßlichen Blutungsquelle angelegt werden. So sind Zugänge der unteren Extremität (i.v. oder i. o.) bei V.a. Abdominal-/Beckentrauma kontraindiziert und sollten an der oberen Extremität angelegt werden. Zur Volumentherapie bei Traumapatienten sollten bevorzugt balancierte kristalloide, isotonische Vollelektrolytlösungen eingesetzt werden [5]. Isotonische Kochsalzlösungen und Humanalbumin haben in der präklinischen Volumentherapie keinen Stellenwert [5]. Hypertonische Lösungen können beim polytraumatisierten Patienten nach stumpfem Trauma mit hypotonischen Kreislaufverhältnissen, bei penetrierendem Trauma sowie hypotonischen Patienten mit schwerem SHT gegeben werden [6]. Kolloidale Lösung sollten nur unter strenger Indikations- und Dosisbeachtung eingesetzt werden, da komplikative Verläufe und eine iatrogene Koagulopathie beschrieben wurden [5]. Die Inzidenz der traumatisch bedingten Koagulopathie wird in Abhängigkeit von der Verletzungsschwere und -art mit bis zu 50\% angegeben. Diesbezüglich hat eine frühzeitige Gabe von Tranexamsäure eine signifikante Reduzierung der Gesamtmortalität blutender Traumapatienten zur Folge, da die auftretende Hyperfibrinolyse im Zuge der Koagulopathie unterbunden wird [22]. Präklinisch sollte nur bei Patienten mit schwerem SHT mit/ohne Multisystemverletzung und V.a. innere Blutung/nicht kompressible Blutung mit hämodynamischer Instabilität $1 \mathrm{~g}$ Tranexamsäure i.v. verabreicht werden, da Studien auch eine erhöhte Rate an thrombembolischen Komplikationen bei nicht fachgerechter Anwendung nachgewiesen haben [23].

Weiterhin gibt es vielversprechende Studien der präklinischen Gabe von Blutprodukten bei Patienten mit nicht kompressiblen inneren Blutungen, sodass hier in Zukunft ggf. Notfallerythrozytenkonzentrate und Plasmen auf den notarztbesetzten Einsatzmitteln mitgeführt werden könnten [24, 25].

Solange Notfallblutprodukte nicht mitgeführt werden, ist vor allem in Situationen von eingeklemmten Personen mit mutmaßlichen inneren Blutungen und langer technischer Rettungszeit (z. B. Lkw-Auffahrunfall) das Verbringen von ungekreuzten Notfallkonserven aus einer benachbarten Klinik an den Unfallort einsatztaktisch zu erwägen. Äußere Blutungen können im Rahmen der körperlichen Untersuchung nachgewiesen werden und sind häufig durch den Einsatz von einfachen Druckverbänden zu stillen. Im Sinne eines Stufenschemas kommt die Anlage eines Tourniquets als Option bei Versagen anderer Mechanismen (Druckverband, Hochlagerung der Extremität) zur Blutstillung als letzte Option infrage [5]. Die Verwendung von lokal wirkenden Hämostyptika kann 
bei vorangegangenen, nicht erfolgreichen Maßnahmen ergänzt werden, wobei hier die Studienlage sehr limitiert zu einer Empfehlung drängt [5].

Eine Sonderform in der Präklinik stellen durch Beckenringfrakturen bedingte Blutungen dar. Trotz Limitationen der körperlichen Untersuchung (siehe oben) soll das Becken klinisch auf seine Stabilität hin untersucht werden. Bei Verdacht auf eine Beckenringverletzung ist die nicht invasive externe Stabilisierung des Beckenrings eine effektive Methode, um spongiöse und venöse Blutungen einzudämmen und sollte angelegt werden, wobei eine Überkompression zu vermeiden ist (manuelle Kompression). Wenn aufgrund des Unfallmechanismus der Verdacht einer Beckenringverletzung nicht ausgeschlossen ist, sollte auch bei hämodynamisch stabilen Patienten eine sofortige Stabilisierung mit einem Beckengurt präklinisch erfolgen [6]. Die Idee dahinter liegt im Effekt der Verringerung der Blutung pro Zeiteinheit durch Anstieg des intrapelvinen Druckes und der Volumenverkleinerung des intrapelvinen Volumens. Hierzu können präklinisch Tuchschlingen oder auch entsprechend konzipierte externe Beckenstabilisatoren $\left(\right.$ T-POD $^{\circledR}$, Sam-Sling ${ }^{\circledR}$, pneumatische Beckenschlinge VBM) verwendet werden. In einer Studie der Autoren in Kooperation mit der Rechtsmedizin der Charité - Berlin (PD Dr. med. C. Buschmann) konnte für alle nicht invasiven externen Beckenstabilisatoren ein Effekt auf die Verringerung des intrapelvinen Volumens nachgewiesen werden, wenngleich der T-POD und die VBM-Schlinge mit $200 \mathrm{mmHg}$ Druck die besten Ergebnisse lieferten. Für eine optimale biomechanische Stabilität sollte nach Innenrotation der Beine die Anlage der Beckenstabilisatoren über den Trochanteres der Oberschenkelknochen erfolgen. Die Überprüfung der pDMS (periphere Durchblutung, Motorik, Sensibilität) nach Anlage eines externen Beckenstabilisators ist obligat [6].

\section{D: Neurotrauma}

Im Wesentlichen handelt es sich hierbei um SHT und Wirbelsäulenverletzungen. Nach Überprüfung und Sicherung der Vitalfunktionen beim ansprechbaren Patienten beinhaltet die Untersuchung der Wirbelsäule am Unfallort die orientierende neurologische Untersuchung bez. der Sensibilität und der Motorik. Zur präklinischen Einschätzung eines SHT ist die wiederholte Erfassung und Dokumentation der Bewusstseinslage, Pupillenfunktion und GCS entscheidend [26].

Eine gezielte körperliche Untersuchung, inklusive der Wirbelsäule und der mit ihr verbundenen Funktionen ist essenziell und erfolgt im Rahmen der körperlichen Untersuchung. Beim bewusstlosen Traumapatienten soll bis zum Beweis des Gegenteils von einer Wirbelsäulenverletzung ausgegangen werden. Hinweise auf die Wahrscheinlichkeit kann die Bewertung des zugrunde liegenden Unfallmechanismus geben. Bei Stürzen aus großer Höhe und Hochgeschwindigkeitsunfällen ist das Risiko erhöht. Ein neurologisches Defizit kann auf das Vorliegen einer Rückenmarksverletzung hinweisen. Das Ausbleiben von Rückenschmerzen ist kein Ausschlusskriterium für eine relevante Wirbelsäulenverletzung. Die Inspektion (Verletzungszeichen/Deformitäten) und das Abtasten (Druck-/ Klopfschmerz oder Stufen/tastbare Lücken zwischen den Dornfortsätzen) der Wirbelsäule vervollständigen die Untersuchung. Wichtig für die weitere Therapie in der Klinik ist die neurologische Funktion der Extremitäten, sodass bei Patienten mit V.a. Wirbelsäulenverletzung wann immer möglich vor Notfallnarkose eine grob orientierende neurologische Untersuchung durchgeführt werden soll. Die Immobilisierung der Halswirbelsäule durch manuellen Handgriff bzw. mit einer entsprechenden Zervikalstütze sollte als erste präklinische Maßnahme unverzüglich erfolgen und wurde deshalb der Atemwegssicherung (siehe A) zugeordnet. Allerdings besteht eine Restbeweglichkeit der HWS, die durch Lagerung auf einer Vakuummatratze effektiv behoben werden kann. Weiterhin ist auch die Zervikalorthese mit einer venösen Kongestion assoziiert, die bei Patienten mit schwerem SHT zu Hirndruckproblematiken führen kann. Dennoch sollte bei allen Patienten mit SHT aufgrund der Koinzidenz von HWS-Verletzungen in der Präklinik eine Orthese angelegt werden. Die Immobilisation der BWS/LWS erfolgt mittels Vakuummatratze oder Spineboard. Zur Umlagerung oder Rettung aus dem Gefahrenbereich haben sich hier die Schaufeltrage und das KED ${ }^{\circledR}$-System bewährt [6].

Da eine kausale Therapie präklinisch nicht möglich ist, handelt es sich um eine rein symptomatische Therapie. Hierbei haben sich die Aufrechterhaltung der zerebralen und spinalen Perfusion und die Vermeidung einer Hypoxie als entscheidend herausgestellt. Beim Erwachsenen sollte eine arterielle Normotension mit einem systolischen Blutdruck nicht unter $90 \mathrm{mmHg}$ (altersadaptiert bei Kindern) angestrebt werden. Hirnprotektive Maßnahmen beinhalten eine ausreichende Oxygenierung $\left(\mathrm{SpO}_{2}\right.$ $>90 \%$ ), Normoventilation (etCO $235-45 \mathrm{mmHg}$ ) und Kreislaufstabilisierung [6]. Die präklinisch eingeleitete Kortisontherapie entsprechend dem NASCIS-Schema (NASCIS: National Acute Spinal Cord Injury Study) ist für SHT-Patienten kontraindiziert und bei Wirbelsäulenverletzungen mit neurologischem Defizit sehr umstritten [27]. Bei Patienten mit schwerem SHT und Symptomen einer transtentoriellen Herniation (Mydriasis, Strecksynergismen, fortschreitende Bewusstseinstrübung, Streckreiz auf Schmerzreaktion) kann die milde Hyperventilation (etCO $230-35 \mathrm{mmHg}$ ) als frühzeitige, hirndrucksenkende Behandlungsmaßnahme nach erfolgtem Trauma eingesetzt werden. Eine zu aggressive Hyperventilation ( etCO $_{2}$ $<30 \mathrm{mmHg}$ ) kann durch Vasokonstriktion zur zerebralen Minderperfusion führen. Weitere Maßnahmen, falls vorhanden, stellen die präklinischen Gaben von Mannitol (20\% $125 \mathrm{ml}$ als Bolus) und hypertonischen Kochsalzlösungen dar. 
Als Lagerungsmaßnahme bei mutmaßlichem Hirndruck empfiehlt sich die $30^{\circ}$-Oberkörperhochlagerung, wodurch eine Senkung des intrakraniellen Druckes erzielt werden kann $[6,28]$.

Bei Patienten mit neurologischen Ausfällen bzw. Verdacht auf eine vorliegende Wirbelsäulenverletzung sollte der Transport in ein geeignetes Traumazentrum mit neuro- und wirbelsäulenchirurgischer Expertise möglichst schonend erfolgen, weshalb bevorzugt die Luftrettung zum Einsatz kommen sollte.

\section{E: Frakturen und Weichteilmanagement}

Gemäß der aktuellen S3-Leitlinie Polytrauma hat die Versorgung von stark blutenden Verletzungen der Extremitäten, welche die Vitalfunktion beeinträchtigen, eine hohe Priorität (siehe C).

Präklinisch sollten alle Extremitäten eines Verunfallten orientierend untersucht werden. Hierbei spielt die Entkleidung des Patienten eine wesentliche Rolle, um eventuell lebensbedrohliche Blutungsquellen nicht zu übersehen. Dies ist vor allem bei penetrierenden Verletzungen essenziell wichtig. Ebenso sollte der Rücken des Patienten, am besten im Rahmen von Umlagerungsmaßnahmen auf die Trage, inspiziert werden. Eine auch nur vermutlich verletzte Extremität sollte vor grober Bewegung/dem Transport des Patienten ruhiggestellt werden.

Weitere Schäden können durch eine frühzeitige Versorgung von Verletzungen der Extremitäten vermieden werden. Dabei sollten Maßnahmen zur Extremitätenversorgung bei instabilen Patienten nicht die Gesamtrettungszeit verzögern [6].

Fehlstellungen und Luxationen der Extremitäten sollten retiniert werden, insbesondere bei begleitender Ischämie der betroffenen Extremität und langer Rettungszeit [6]. Zur Immobilisation von Extremitäten kommen vor allem Schienen, Extensionsschienen (Braun, Kirschner, Krapp, Kendric Traction Device), SamSplint ${ }^{\circledR}$ und Dreieckstücher zum Einsatz [9].

Bezüglich des Schienungsmaterials ist im Wesentlichen zwischen Vakuumsystem und Luftkammersystemen zu unterscheiden. Vorteil der Vakuumschienen ist die gute Anmodulierbarkeit und Kontrollierbarkeit des externen Druckes. Der Vorteil der Luftkammerschienen ist ein zusätzlicher kompressiver Effekt vor allem bei offenen Frakturen und Weichteilverletzungen, die auf Kosten eines zusätzlichen Weichteiltraumas eine Blutstillung zur Folge haben. Offene Frakturen sollen von groben Verschmutzungen gereinigt (Ausspülen mit isotonischer Infusionslösung) und steril verbunden werden. Hier hat sich im eigenen Vorgehen das Mitführen von sterilen Bauchtüchern bewährt [6].
Bei schweren Verletzungen mit Amputation sollte abgesehen von einer Blutstillung der Amputationsstumpf geschient und steril verbunden werden. Das Amputat ist zu asservieren und in sterile feuchte Kompressen einzuwickeln. Zur Kühlung sollte die „Doppelbeutelmethode“ angewendet werden, wobei direkter Kontakt des Kühlmittels mit dem Amputat strikt vermieden werden muss. Dabei wird das Amputat in einen inneren Plastikbeutel mit sterilen, feuchten Kompressen verpackt. Anschließend wird dieser Beutel in einen weiteren Beutel mit Eiswasser (1/3 Eiswürfel, 2/3 Wasser) gelegt und verschlossen. Herausgeschlagene Zähne und Zahnfragmente sollen ebenso aufgenommen, feucht gelagert und zur Replantation ins Traumazentrum mitgebracht werden. Bei perforierenden Verletzungen sollte der perforierende Gegenstand belassen und gepolstert werden ( $\bullet$ Abb. $\mathbf{3}$ ).

Bei eingeklemmten Extremitäten und instabilen Patienten, die nicht zeitgerecht gerettet werden können, ist eine Notamputation zu erwägen. Da dies nicht von jedem Notarzt erwartet werden kann, jedoch in Deutschland nahezu in allen Gegenden in 20 min Entfernung eine Klinik mit chirurgischer Abteilung vorgehalten wird, muss einsatztaktisch überlegt werden, ein Notamputationsteam mit Equipment an die Einsatzstelle zu bringen [6].

Der Wärmeerhalt spielt ebenso eine große Rolle, daher müssen während der Rettung des Patienten sowie auch zum Zeitpunkt der klinischen Untersuchung bis hin zum Transport in die Zielklinik Maßnahmen zum Schutz vor dem Auskühlen getroffen werden. Die akzidentielle entstehende Hypothermie hat negative Auswirkungen auf das Gerinnungssystem und insgesamt auf das spätere Outcome. Daher sollte frühzeitig auf einen suffizienten Wärmeerhalt (gewärmte Infusionslösungen, Wärmedecke, Aluminiumfolie) geachtet werden [6].

In der nachfolgenden Infobox sind die wesentlichen präklinischen Maßnahmen dem ABCDE-Schema entsprechend aufgeführt.

\section{Traumatische Reanimation}

Die Weiterentwicklung des Traumamanagements führte zu einer differenzierteren Analyse der pathophysiologischen Probleme. Diese unterscheiden sich grundlegend von kardiovaskulär bedingten Herz-Kreislauf-Stillständen, wobei ohne kausale Therapie des obstruktiven Schocks (Dekompression Spannungspneumothorax, Perikardtamponade) es zu keinem Wiedereintritt des Kreislaufes (ROSC) kommt.

Der vor 20 Jahren noch als aussichtslose Situation beschriebene traumatische Herz-Kreislauf-Stillstand wird heute nach potenziell reversiblen Ursachen hin behandelt, sodass Überlebensraten bis über $20 \%$ beschrieben wurden [29]. Potenziell reversible Ursachen des traumati- 


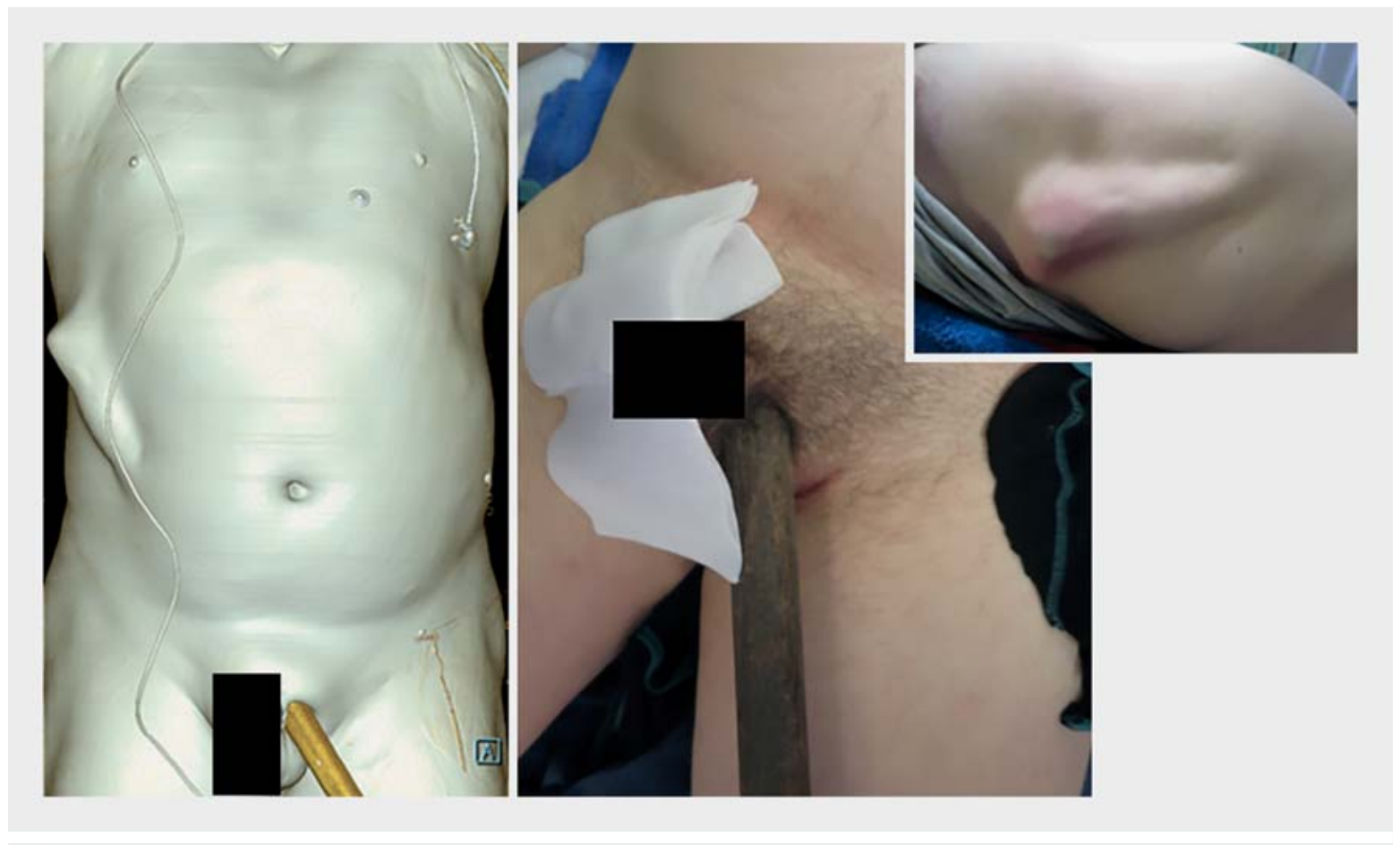

Abb. 3 Fall eines 47-jährigen Mannes mit Pfählungsverletzung: Belassung des Fremdkörpers und Polsterung bis in den Operationssaal. Quellen: a Institut und Poliklinik für Diagnostische und Interventionelle Radiologie, Universitätsklinikum Carl Gustav Carus Dresden.

\section{INFOBOX}

A: Atemwege sichern (Intubation, alternativer Atemweg, Cricothyroidotomie, Koniotomie, Tracheotomie), HWS-Immobilisation

B: Sicherung Ventilation (Beatmung, Dekompression Spannungspneumothorax, Pleurasiegel) und Oxygenierung $\left(\mathrm{O}_{2}\right.$-Gabe, Beatmung)

C: Blutungskontrolle (Druckverband, Tourniquet, Hämostyptikum), Aufrechterhaltung Kreislauf (Volumensubstitution, permissive Hypotonie, Katecholamine), Blutgerinnung (Tranexamsäure), Beckenstabilisation, (Blutprodukte), Perikardiozentese/Notfallthorakotomie

D: Immobilisation, ggf. Hirndrucktherapie (Hyperventilation, Mannitol, hypertonische Lösungen)

E: Wärmeerhalt, Reposition, Wundversorgung, Ausspülen offener Frakturen, Notfallamputation

schen Herz-Kreislauf-Stillstands sind dabei die $4 \times \mathrm{H}$ und HITS ( $\triangleright$ Tab. 4):

Unter Berücksichtigung der definitiv und potenziell vermeidbaren Todesursachen führte dies zur Entwicklung des ersten präklinischen Reanimationsalgorithmus für Traumapatienten. Ein Fall der Autoren mit primär erfolg-
- Tab. 4 Ursachen des traumatischen Herz-Kreislauf-Stillstands: $4 \times \mathrm{H}$ und HITS.

\begin{tabular}{|l|l|}
\hline Hypoxie & Herzbeuteltamponade \\
\hline Hypovolämie & Intoxikation \\
\hline Hypothermie & Thrombembolie \\
\hline Hypo-/Hyperkaliämie & Spannungspneumothorax \\
\hline
\end{tabular}

reicher präklinischer Reanimation eines Patienten mit Aortenruptur nach Auffahrunfall unterstreicht die Potenz und Effektivität aller unter ABCDE genannten Maßnahmen, die in einem Algorithmus angewendet werden ( $\bullet$ Abb. 4) [30].

Basismaßnahme ist wie bei allen Kreislaufstillständen die externe Thoraxkompression und Beatmung (30:2). Diese sind bei fehlenden Lebenszeichen, bei Unsicherheiten im Nachweis eines Pulses oder anderen klinischen Zeichen, die einen Herz-Kreislauf-Stillstand wahrscheinlich machen, unverzüglich durchzuführen. Im Zuge der kardiopulmonalen Reanimation sollen leitliniengerecht traumaspezifische reversible Ursachen des Herz-Kreislauf-Stillstands (nach ABCDE-Schema; z.B. Atemwegsobstruktionen, ösophageale Fehlintubation, Spannungspneumothorax, Perikardtamponade und Hypovolämie) diagnostiziert, 


\begin{tabular}{l} 
Hypoxie \\
Spannungspneumothorax \\
Perikardtamponade \\
Hypovolämie \\
\hline
\end{tabular}

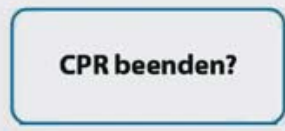

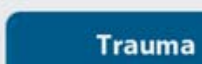

Patient
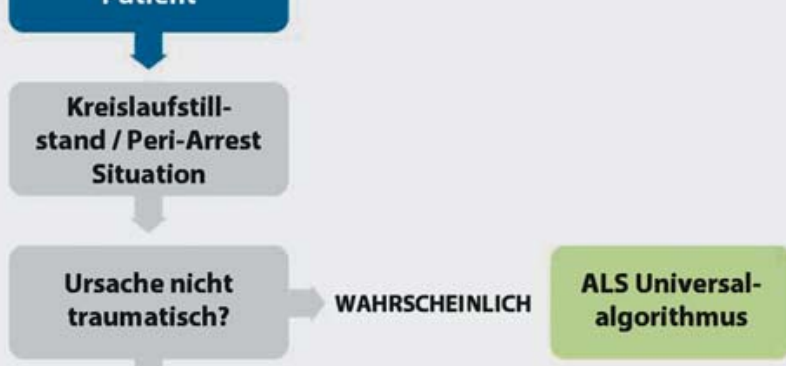

UNWAHRSCHEINLICH

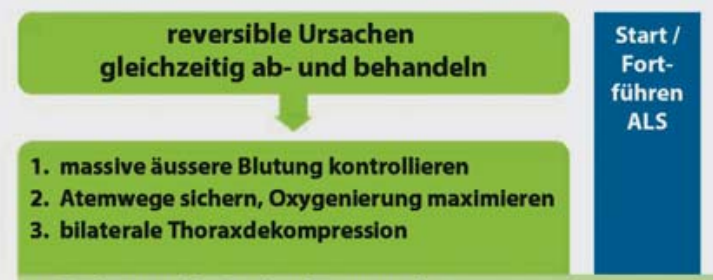

\section{Entlastung der Perikardtamponade \\ 5. chirurgische Blutungskontrolle oder proximale Aortenkompression}

6. Flüssigkeitstherapie und

Massivtransfusionsprotokoll NEIN

\section{Wiederkehr des} Spontankreislaufs
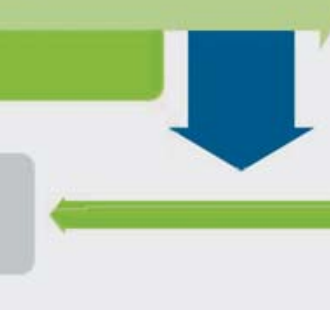

Elapsed time/Verzögerung $<10$ min nach Kreislaufstillstand? Expertise? / Erfahrung? Equipment? / Ausrüstung? Enviroment?/Umgebung?

Notfallthorakotomie in Erwägung ziehen

JA

präkllnisch:
- nur lebensrettende Massnahmen durchführen
- schnellstmöglicher Transport In eine KIInlk
kllnlsch:
- damage control resuscitation
- definitive Blutungskontrolle

Abb. 4 Algorithmus zur Behandlung eines traumatischen Herz-Kreislauf-Stillstands. Quelle: Truhláŕ A, Deakin CD, Soar J et al. Kreislaufstillstand in besonderen Situationen. Kapitel 4 der Leitlinien zur Reanimation 2015 des European Resuscitation Council. Notfall Rettungsmed 2015; 18: 883-903. @ German Resuscitation Council (GRC) und Austrian Resuscitation Council (ARC) 2015. [rerif]

ausgeschlossen und/oder prioritär therapiert werden [6]. Dabei sind dekompressive Maßnahmen bei Spannungspneumothorax und Perikardtamponade prioritär durchzuführen, da eine externe Herzdruckmassage ohne passive Füllung des Herzens ineffektiv ist. Dies unterstreicht eine Studie, die einen signifikanten Überlebensvorteil bei beidseitiger Entlastung mittels Minithorakotomie nachweisen konnte [6,31].

Liegt ein Kreislaufstillstand nach penetrierendem Thoraxtrauma vor, ist bei gegebenen Voraussetzungen die Indi- kation für eine Nadelperikardiozentese oder bei optimalen Rahmenbedingungen und Ausbildung des Notarztes ggf. auch präklinische Notfallthorakotomie („Clamshell“Zugang) gegeben [32]. Klinische Zeichen einer Perikardtamponade ähneln der des Spannungspneumothoraxes und sind:

- Unfallmechanismus, vor allem penetrierende Verletzung in der „cardiac box“

- obere Einflussstauung (Halsvenen)

- Niedervoltage im EKG

- sicherer Nachweis im eFAST 


\section{Zusammenfassung}

Die optimale Versorgung von schwerverletzten Patienten in der Frühphase des Traumas erfordert nach wie vor ein zeitkritisches Handeln. Bei der Entscheidung der Einsatztaktik ist im Rahmen der präklinischen Versorgung die Dringlichkeit der erfordernden notärztlichen Maßnahmen und deren Therapierbarkeit (Spannungspneumothorax vs. innere Blutung) ausschlaggebend [33-35]. Anlässlich der Komplexität eines Polytraumas mit multiplen Verletzungskombinationen und teilweise simultan lebensbedrohlichen Problemen hat sich das ABCDE-Schema der Notfallmedizin als Grundlage der präklinischen Polytraumaversorgung ( $\mathrm{pHTLS}^{\circledR}, \mathrm{ETC}^{\circledR}$, TraumaManagement $^{\circledR}$ ) durchgesetzt. Aufgrund der Notwendigkeit von invasiven präklinischen Maßnahmen muss das notärztliche Personal regelmäßig in der Notfallnarkose, der endotrachealen Intubation, in alternativen Methoden der Atemwegssicherung, Thoraxdrainageanlage, Perikardiozentese, eFAST und Umgang mit externen Beckenstabilisatoren, Schienungsmaterial und Techniken der Blutstillung trainiert werden. Aufgrund eines Überlebensvorteils für die Patienten, weniger vermeidbarer Todesfälle und logistischer Vorteile (Transport in geeignete Zielklinik) sollte die Luftrettung primär zur präklinischen Versorgung von Schwerverletzten eingesetzt werden.

\section{Interessenkonflikt}

Die Autoren geben an, dass kein Interessenkonflikt besteht.

\section{Autorinnen/Autoren}

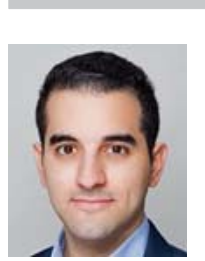

\section{Delovan Oramary}

Dr. med., univ. Assistenzarzt, UniversitätsCentrum für Orthopädie und Unfallchirurgie (OUC), Universitätsklinikum Carl Gustav Carus, TU Dresden
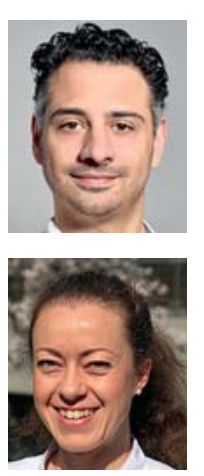

\section{Onays Al-Sadi}

Dr. med., Assistenzarzt, UniversitätsCentrum für Orthopädie und Unfallchirurgie (OUC), Universitätsklinikum Carl Gustav Carus, TU Dresden

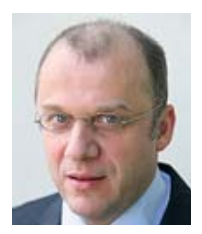

\section{Sophia Blum}

Dr. med., Funktionsoberärztin, Institut und Poliklinik für diagnostische und interventionelle Radiologie, Universitätsklinikum Carl Gustav Carus, TU Dresden

\section{Klaus Dieter Schase}

Univ.-Prof. Dr. med., Ärztlicher Direktor, UniversitätsCentrum für Orthopädie und Unfallchirurgie (OUC), Universitätsklinikum Carl Gustav Carus, TU Dresden

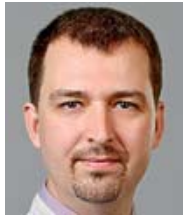

\section{Christian Kleber}

PD Dr. med., Geschäftsführender Oberarzt, Ärztlicher Leiter Chirurgische Notaufnahme, Stellv. Leitender Arzt Christoph 38, UniversitätsCentrum für Orthopädie und Unfallchirurgie (OUC), Universitätsklinikum Carl Gustav Carus, TU Dresden; Christoph 38 Station Dresden, Deutsche Rettungsflugwacht (DRF)

Korrespondenzadresse

PD Dr. med. Christian Kleber

UniversitätsCentrum für Orthopädie und Unfallchirurgie (OUC)

Universitätsklinikum Carl Gustav Carus, TU Dresden

Fetscherstraße 74

01307 Dresden

christian.kleber@ukdd.de

\section{Literatur}

[1] Kleber C, Giesecke MT, Tsokos M et al. Trauma-related preventable deaths in Berlin 2010: need to change prehospital management strategies and trauma management education. World J Surg 2013; 37: 1154-1161

[2] Kleber C, Lefering R, Kleber AJ et al. [Rescue time and survival of severely injured patients in Germany]. Unfallchirurg 2013; 116: $345-350$

[3] Sektion Intensiv- \& Notfallmedizin, Schwerverletztenversorgung der Deutschen Gesellschaft für Unfallchirurgie e.V.; AUC - Akademie der Unfallchirurgie GmbH. Traumaregister DGU Jahresbericht 2018. Im Internet: http://www.traumaregisterdgu.de/fileadmin/user_upload/traumaregister-dgu.de/docs/ Downloads/TR-DGU-Jahresbericht_2018.pdf; Stand: 16.10.2019

[4] Ondruschka B, Baier C, Dressler ] et al. [Additional emergency medical measures in trauma-associated cardiac arrest]. Anaesthesist 2017; 66: 924-935

[5] Bundesärztekammer. Indikationskatalog der Bundesärztekammer für den Notarzteinsatz, Stand: 22.02.2013. Im Internet: https://www.bundesaerztekammer.de/fileadmin/ user_upload/downloads/NAIK-Indikationskatalog_fuer_den_ Notarzteinsatz_22022013.pdf; Stand: 16.10.2019

[6] Arbeitsgemeinschaft der Wissenschaftlichen Medizinischen Fachgesellschaften e.V. (AWMF). S3-Leitlinie Polytrauma/ Schwerverletztenbehandlung der DGU. Stand: 07/2016. Im Internet: https://www.awmf.org/uploads/tx_szleitlinien/012019l_S3_Polytrauma_Schwerverletzten-Behandlung_201708.pdf; Stand: 16.10.2019

[7] Pape H, Hildebrand F, Ruchholtz S. Management des Schwerverletzten. Heidelberg: Springer; 2018

[8] Keel M, Trentz O. Pathophysiology of polytrauma. Injury 2005; 36: 691-709

[9] Kleber C, Lindner T, Bail H. Erstversorgung von Frakturen und Luxationen. Notfall Rettungsmed 2009; 12: 551-560

[10] Byard RW. How reliable is external examination in identifying internal injuries - Casper's sign revisited. J Forensic Leg Med 2012; 19: 419-421

[11] Frank M, Schmucker U, Stengel D et al. Proper estimation of blood loss on scene of trauma: tool or tale? J Trauma 2010; 69: 1191-1195 
[12] Aufmkolk M, Ruchholtz S, Hering M et al. [The value of subjective estimation of the severity of thoracic injuries by the emergency surgeon]. Unfallchirurg 2003; 106: 746-753

[13] Gonzalez RP, Fried PQ, Bukhalo M. The utility of clinical examination in screening for pelvic fractures in blunt trauma. J Am Coll Surg 2002; 194: 121-125

[14] Pehle B, Nast-Kolb D, Oberbeck R. Wertigkeit der körperlichen und radiologischen Basisdiagnostik des Beckens in der Schockraumbehandlung. Unfallchirurg 2003; 106: 642-648

[15] Dunham CM, Barraco RD, Clark DE et al. Guidelines for emergency tracheal intubation immediately after traumatic injury. J Trauma 2003; 55: 162-179

[16] Nolan JP, Deakin CD, Soar J et al. European Resuscitation Council guidelines for resuscitation 2005. Section 4. Adult advanced life support. Resuscitation 2005; 67 (Suppl. 1): S39-S86

[17] Braun P, Wenzel V, Paal P. Anesthesia in prehospital emergencies and in the emergency department. Curr Opin Anaesthesiol 2010; 23: 500-506

[18] Lockey D, Crewdson K, Weaver A et al. Observational study of the success rates of intubation and failed intubation airway rescue techniques in 7256 attempted intubations of trauma patients by pre-hospital physicians. Br J Anaesth 2014; 113: $220-225$

[19] Helm M, Hossfeld B, Jost C. Chirurgische Atemwegssicherung in der präklinischen Notfallmedizin. Notfall Rettungsmed 2011; 14: 29-36

[20] Bernard SA, Nguyen V, Cameron P et al. Prehospital rapid sequence intubation improves functional outcome for patients with severe traumatic brain injury: a randomized controlled trial. Ann Surg 2010; 252: 959-965

[21] Quehl E, Manthey M, Giesecke M et al. Management eines offenen Pneumothorax mit intrathorakalem Fremdkörper im Rahmen schizophrener Wahnvorstellungen. Notfall Rettungsmed 2012; 15: 423-428

[22] Morrison J], Dubose J], Rasmussen TE et al. Military Application of Tranexamic Acid in Trauma Emergency Resuscitation (MATTERs) Study. Arch Surg 2012; 147: 113-119

[23] Lier H, Maegele M. Blutungsmanagement: Tranexamsäure in der Präklinik. Pro und Kontra. Notfall Rettungsmed 2018; 21: 1-11

[24] Shackelford SA, Del Junco DJ, Powell-Dunford N et al. Association of prehospital blood product transfusion during medical evacuation of combat casualties in Afghanistan with acute and 30-day survival. JAMA 2017; 318: 1581-1591
[25] Sperry JL, Guyette FX, Brown JB et al. Prehospital plasma during air medical transport in trauma patients at risk for hemorrhagic shock. N Engl J Med 2018; 379: 315-326

[26] Gabriel EJ, Ghajar J, Jagoda A et al. Guidelines for prehospital management of traumatic brain injury. J Neurotrauma 2002; 19: $111-174$

[27] Bernhard M, Gries A, Kremer P et al. Spinal cord injury (SCI)prehospital management. Resuscitation 2005; 66: 127-139

[28] Brain Trauma Foundation; American Association of Neurological Surgeons; Congress of Neurological Surgeons. Guidelines for the management of severe traumatic brain injury. J Neurotrauma 2007; 24 (Suppl. 1): S1-S106

[29] Buschmann C, Tsokos M, Kleber C. Vermeidbare Todesfälle nach Trauma. Notfall Rettungsmed 2015; 18: 316-324

[30] Kleber C. Primarily successful resuscitation of traumatic aortic rupture after high-energy car accident: a case report. Emergency Med 2018; 8: 371

[31] Bushby N, Fitzgerald M, Cameron P et al. Prehospital intubation and chest decompression is associated with unexpected survival in major thoracic blunt trauma. Emerg Med Australas 2005; 17: 443-449

[32] Truhláŕ A, Deakin CD, Soar J et al. European Resuscitation Council Guidelines for Resuscitation 2015: Section 4. Cardiac arrest in special circumstances. Resuscitation 2015; 95: 148 201

[33] Hossfeld B, Lechner R, Josse F et al. [Prehospital application of tourniquets for life-threatening extremity hemorrhage: systematic review of literature]. Unfallchirurg. 2018; 121: 516529

[34] Kulla M, Helm M, Lefering R et al. Prehospital endotracheal intubation and chest tubing does not prolong the overall resuscitation time of severely injured patients: a retrospective, multicentre study of the Trauma Registry of the German Society of Trauma Surgery. Emerg Med J 2012; 29: 497-501

[35] Kleber C, Giesecke M, Buschmann C. Overall distribution of trauma-related deaths in Berlin 2010: the weakest links of the chain of survival are emergency medicine and critical care: reply. World J Surg 2013; 37: 475

\section{Bibliografie}

DOI https://doi.org/10.1055/a-0990-2170

Online-publiziert 27.11.2019 | OP-JOURNAL 2020; 36: 5-16 (c) Georg Thieme Verlag KG Stuttgart · New York ISSN 0178-1715 